\title{
Attitude control of VTOL-UAVs.
}

\author{
HEIDARIAN, M. and MEMON, A.Y.
}

2012

(C) 2012 IEEE. Personal use of this material is permitted. Permission from IEEE must be obtained for all other uses, in any current or future media, including reprinting/republishing this material for advertising or promotional purposes, creating new collective works, for resale or redistribution to servers or lists, or reuse of any copyrighted component of this work in other works. 


\title{
Attitude Control of VTOL-UAVs
}

\author{
Maryam Heidarian ${ }^{\dagger}$ and Attaullah Y. Memon ${ }^{\ddagger}$
}

\begin{abstract}
This paper presents a novel control approach to obtain asymptotic attitude stability of a quadrotor as a representative of Planar Vertical Take Off and Landing (PVTOL) Unmanned Aerial Vehicles (UAVs). The considered quadrotor is a symmetric VTOL-UAV with four rigid mono-directional propellers, which has been modeled based on quaternion representation with taking Coriolis and gyroscopic torques into account. In the proposed approach, two nearly equivalent control laws (model independent as well as model dependent) have been used to obtain exponential stability of attitude angles and asymptotic stability of attitude angular velocity of the quadrotor UAV. The proposed approach also presents how the attitude parameters i.e. attitude angles and attitude angular velocity can be quickly regulated to their desired values as required.
\end{abstract}

Index Terms-Attitude Control, Velocity Control, VTOL, UAV, Quadrotor.

\section{INTRODUCTION}

T is more than two decades since the first VTOL-UAVs were fully demonstrated in practical research works. VTOLUAVs constitute the class of Unmanned Aerial Vehicles which have the ability of Vertical Take Off and Landing and thus have the capability of fast target acquisition. Due to this, their applications have found a growing interest in performing certain tasks which require high maneuverability and robustness with respect to unknown external disturbances. These UAVs can be used both as individual vehicles and in a team formation of multiple vehicles [1]. Furthermore, use of VTOL-UAVs has been envisaged in a variety of applications, e.g. in environmental protection, intervention in hostile sites, natural risk management, remote inspections, rescue missions, agriculture and, commercial video production. More recently, small quadrotor UAVs have attracted considerable attention by researchers, due to its less complicated mechanical design and maintenance aspects in comparison to helicopters. A quadrotor is essentially a helicopter which has four propellers in cross configuration.

In order to effectively control a VTOL-UAV, a composite control scheme comprising of two different controllers is required, namely: the attitude controller and the position controller. Design of these two controllers constitutes challenging tasks and the same have been addressed separately in the literature. In [2], a dynamical model of quadrotor based on quaternion representation has been derived from NewtonEuler equations. Hamel et al., in [3], identify dynamics of the vehicle beyond the basic nonlinear equations of

\footnotetext{
$\dagger \ddagger$ The authors are with the Department of Electronics and Power Engineering, PN Engineering College, National University of Sciences \& Technology (NUST), Karachi, Pakistan.

(E-mail: maryamh@pnec.edu.pk, attaullah@pnec.edu.pk)
}

motion, with gyroscopic torque and Coriolis terms. Based on this model, Tayebi and McGilvray [4], have represented a model independent $P D$ controller with asymptotic stability and a model dependent $P D^{2}$ controller with exponential stability. A method to obtain attitude control stabilization of a quadrotor through using backstepping technique and adding saturation functions has been analyzed in [5]. Precise measurement of the angular velocity and the initial orientation are required for attitude stabilization of these vehicles. Due to various uncertainties (related to gyroscope and other effects), there may be some errors in these measurements. Using inertial measurements units (IMU's) information to estimate these required values is one of the possible ways to reduce the errors [6]. In their more recent work [7], Tayebi et al., have considered control designs that do not necessarily require exact knowledge of the angular velocity of the aerial vehicle.

In this paper, we consider two nearly equivalent control laws (model independent as well as model dependent) to obtain exponential stability of attitude angles and asymptotic stability of attitude angular velocity of the quadrotor UAV. The rest of the paper is organized as follows. Section II states the problem formulation and presents the necessary mathematical foundation. Section III presents the control design and main results. The simulation results are presented in Section IV, and finally Section V draws the conclusions.

\section{Problem Formulation}

A quadrotor simply consists of four lift generating propellers mounted on motors. These motors are located at the lateral sides of a cross shaped frame with an angle of 90 degrees between the arms. Center of mass is placed at the intersection of the line joining rotors 1 and 3 and the line joining rotors 2 and 4 , which is middle of the connecting links. Furthermore, the quadrotor is assumed to be symmetric and a basic schematic is depicted in Fig. 1.

Flight control of this vehicle is achieved by varying the angular speed of rotors, $w_{i}, 1 \in 1,2,3,4$. For example, pitching is possible with increasing (reducing) the speed of the rear motor while reducing (increasing) the speed of the front motor. This means, the pitch torque is a function of the difference $w_{1}-w_{3}$. Similarly, roll movement is obtained by using the lateral motors. Thus, the roll torque will be a function of $w_{2}-w_{4}$. Similarly, the yaw motion is obtained by increasing (reducing) the speed of the front and rear motors together while reducing (increasing) the speed of the lateral motors together. These movements can be accomplished while keeping the total thrust, $T$, constant. Also the vertical movement is generated by increasing the total thrust. The 


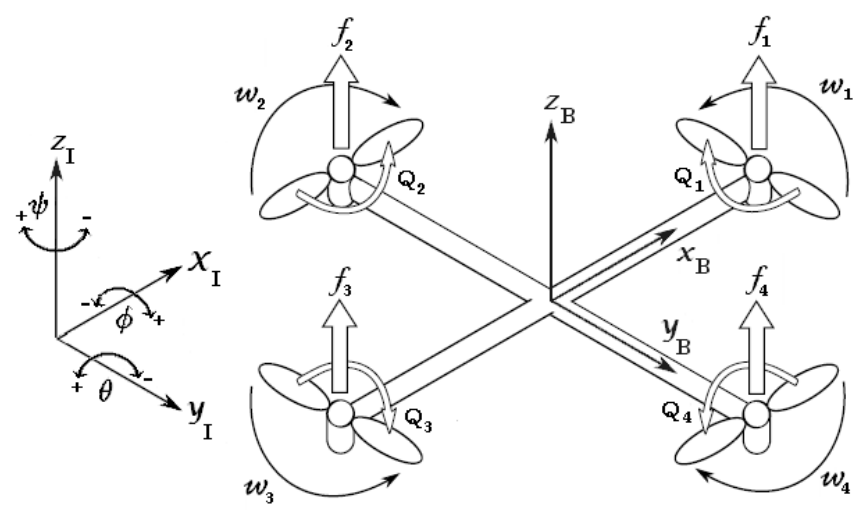

Fig. 1. Illustration of frames

main thrust is expressed as:

$$
\begin{aligned}
& T=\sum_{i=1}^{4}\left|f_{i}\right| \\
& f_{i}=b w_{i}^{2} z_{B}
\end{aligned}
$$

Where $f_{i}$ is the vertically upward lifting force produced by $i$ th motor. As we can see in Fig. 1, the rotation direction of two of the rotors is clockwise while the same for the other two is counterclockwise. This is so to balance the movement of the quad rotor and to prevent any yaw drift caused by the unbalanced reactive torques. The reactive torque of $i$ th rotor is given by

$$
Q_{i}=l w_{i}^{2}
$$

Since, each motor turns in a fixed direction, the produced force $f_{i}$ is always positive. Thus,

$$
T=b \sum_{i=1}^{4} w_{i}^{2}
$$

The constants $l>0$ and $b>0$ in the above two equations are dependent on different aerodynamical parameters [8]. The generalized torques (e.g. roll torque $\tau_{\phi}$, pitch torque $\tau_{\theta}$, and yaw torque $\tau_{\psi}$ ) according to [3] can be represented by

$$
\begin{aligned}
\tau_{\phi} & =b d\left(w_{2}^{2}-w_{4}^{2}\right) \\
\tau_{\theta} & =b d\left(w_{1}^{2}-w_{3}^{2}\right) \\
\tau_{\psi} & =l\left(w_{1}^{2}+w_{3}^{2}-w_{2}^{2}-w_{4}^{2}\right)
\end{aligned}
$$

in which $d$ is the length of arms between the motors and the center of gravity.

It is noteworthy to mention here that a good working knowledge of the quadrotor dynamical model is essential to improve the performance of the aircraft. Simple vector algebraic laws (e.g. commutativity) cannot be applied to finite rotation vectors of rigid bodies. Due to this, we cannot find the attitude of the aircraft from integrating the angular velocities. Dynamical modeling of the quadrotor requires us to define two reference frames: an inertial frame $I$ defined by set of unit vectors $\left\{x_{I}, y_{I}, z_{I}\right\}$ and a body fixed-frame $B$ with orthogonal axes defined by set of unit vectors $\left\{x_{B}, y_{B}, z_{B}\right\}$. In order to define the orientation of the aircraft between these two reference frames, one can use Euler angle description, in which a $3 \times 3$ direction matrix will represent rotation of the aircraft with respect to the body fixed-frame. Euler angle description has an inherent geometric singularity problem. In order to overcome this problem, one can use quaternion representation which defines the rotation of the aircraft with four parameters. The quaternion description is essentially based on Euler's theorem which states that any rotation of an aircraft can be described by a single rotation about a fixed axis.

In what follows, the dynamical model of the quadrotor is obtained via Newton-Euler approach as given in [4]. The basic assumption is that the quadrotor and its propellers are rigid and the external aerodynamic effects (air friction, wind pressure etc.) can be neglected. A simplified model with consideration of Coriolis and gyroscopic torques is given by

$$
\begin{aligned}
\dot{q} & =\frac{1}{2}\left(\begin{array}{c}
-(\bar{q})^{T} \\
s(\bar{q})+q_{0} I
\end{array}\right) \Omega \\
I_{f} \dot{\Omega} & =-s(\Omega) I_{f} \Omega-G_{a}+\tau_{a} \\
I_{r} \dot{w}_{i} & =\tau_{i}-Q_{i} \quad i \in 1,2,3,4
\end{aligned}
$$

where $q$ represents the quaternion equations as given by

$$
\begin{aligned}
q= & \left(\begin{array}{c}
q_{0} \\
\bar{q}
\end{array}\right) \\
= & \left(\begin{array}{c}
\cos \frac{\phi}{2} \cos \frac{\theta}{2} \cos \frac{\psi}{2}+\sin \frac{\phi}{2} \sin \frac{\theta}{2} \sin \frac{\psi}{2} \\
\sin \frac{\phi}{2} \cos \frac{\theta}{2} \cos \frac{\psi}{2}-\cos \frac{\phi}{2} \sin \frac{\theta}{2} \sin \frac{\psi}{2} \\
\cos \frac{\phi}{2} \sin \frac{\theta}{2} \cos \frac{\psi}{2}+\sin \frac{\phi}{2} \cos \frac{\theta}{2} \sin \frac{\psi}{2} \\
\cos \frac{\phi}{2} \cos \frac{\theta}{2} \sin \frac{\psi}{2}-\sin \frac{\phi}{2} \sin \frac{\theta}{2} \cos \frac{\psi}{2}
\end{array}\right)
\end{aligned}
$$

The parameters $\phi, \theta$ and $\psi$ respectively represent the roll, pitch and yaw angular displacements about their related axes, and are defined by the following relations [9];

$$
\begin{aligned}
\dot{\phi} & =\Omega_{\phi}+\left(\Omega_{\theta} \sin \phi+\Omega_{\psi} \cos \phi\right) \tan \theta \\
\dot{\theta} & =\Omega_{\theta} \cos \phi-\Omega_{\psi} \sin \phi \\
\dot{\psi} & =\left(\Omega_{\theta} \sin \phi+\Omega_{\psi} \cos \phi\right) \sec \theta
\end{aligned}
$$

in which $\Omega=\left(\Omega_{\phi}, \Omega_{\theta}, \Omega_{\psi}\right)^{T}$ describes the angular velocity of the quadrotor. Furthermore, $I_{f} \in \mathbb{R}^{3 \times 3}$ is a symmetric positive-definite constant inertia matrix of the airframe with respect to this frame, and $\tau_{a}=\left(\tau_{\phi}, \tau_{\theta}, \tau_{\varphi}\right)^{T}$ denotes the control torque. The notation $I_{r}$ represents the constant moment of inertia of the rotor, and $\tau_{i}, i \in 1,2,3,4$ represents the rotor torques. The function $s(x)$ in (9) represents a skewsymmetric matrix defined as

$$
s(x)=\left(\begin{array}{ccc}
0 & -x_{3} & x_{2} \\
x_{3} & 0 & -x_{1} \\
-x_{2} & x_{1} & 0
\end{array}\right)
$$

and is used to define the term $-s(x) I_{f} \Omega$ in (9), which is due to Coriolis torque. Finally, the term $G_{a}$ denotes the gyroscopic torque and is defined as

$$
G_{a}=I_{r} s(\Omega) z_{I}\left(-w_{1}+w_{2}-w_{3}+w_{4}\right)
$$

in which $z_{I}=(0,0,1)^{T}$. 


\section{Control Design And AnAlysis}

The proposed control algorithm to stabilize the attitude of quadrotor follows closely the approach presented in [4], with some technical differences incorporated to seek for an improvement in overall transient performance. We first design the control torque $\left(\tau_{a}\right)$ that stabilizes the attitude of quadrotor dynamically and then, we synthesize a rotor torque $\left(\tau_{i}\right)$ to obtain the control torque $\left(\tau_{a}\right)$ designed earlier, while considering that the real dynamical input to the quadrotor is angular speed of rotors.

\section{A. Designing the Control Torque $\tau_{a}$}

The dynamical model of the quadrotor, as described in the last section, possesses a cascade structure, in which $\tau_{a}$ controls $\Omega$ and $\Omega$ controls $q$, i.e. $\left(\tau_{a} \rightarrow \Omega \rightarrow q\right)$. This means:

$$
\begin{gathered}
\dot{\Omega}=g\left(\Omega, \tau_{a}\right) \\
\dot{q}=f(q, \Omega)
\end{gathered}
$$

From (8), we have

$$
\begin{gathered}
\dot{q}=f(q, \Omega)=F(\Omega) q \\
F(\Omega)=\frac{1}{2}\left(\begin{array}{cccc}
0 & -\Omega_{\phi} & -\Omega_{\theta} & -\Omega_{\psi} \\
\Omega_{\phi} & 0 & -\Omega_{\psi} & \Omega_{\theta} \\
\Omega_{\theta} & \Omega_{\psi} & 0 & -\Omega_{\phi} \\
\Omega_{\psi} & -\Omega_{\theta} & \Omega_{\phi} & 0
\end{array}\right)
\end{gathered}
$$

Similarly from (9), we have

$$
\dot{\Omega}=g\left(\Omega, \tau_{a}\right)=I_{f}^{-1}\left(-s(\Omega) I_{f} \Omega-G_{a}+\tau_{a}\right)
$$

With this in mind, our goal is to find a suitable control law $\tau_{a}=H(q, \Omega)$. We achieve this objective through the following two steps:

- By finding desired angular velocity $\Omega_{d}=h(q)$ such that when $\Omega_{d}$ is given as input to (9), the solution to the nonlinear equation $\dot{q}=f(q, h(q))$ is asymptotically stable.

- By ensuring that the angular velocity $\Omega$ asymptotically tracks the desired angular velocity $\Omega_{d}$, i.e. $\lim _{t \rightarrow \infty}\left(\sup \left|\Omega-\Omega_{d}\right|\right)=0$.

1) Desired Angular Velocity: The desired angular velocity $\Omega_{d}$, has to be chosen in such a way that the solution of the nonlinear differential equation $\dot{q}=f\left(q, \Omega_{d}\right)$ converges to its equilibrium point. The equilibrium point, with assuming $0 \leq q_{0} \leq 1$ is $q_{e}=(1,0,0,0)^{T}$. The quaternion regulation error can be described by

$$
\widetilde{q}=q-q_{e}=\left(q_{0}-1, q_{1}, q_{2}, q_{3}\right)^{T}
$$

Comparing $\tilde{q}$ with $\dot{q}$ will give us:

$$
\begin{aligned}
\dot{\tilde{q}} & =\frac{1}{2}\left(\begin{array}{c}
-(\bar{q})^{T} \\
s(\bar{q})+\left(q_{0}-1\right) I
\end{array}\right) \Omega \\
& =\frac{1}{2}\left(\begin{array}{ccc}
-q_{1} & -q_{2} & -q_{3} \\
q_{0}-1 & -q_{3} & q_{2} \\
q_{3} & q_{0}-1 & -q_{1} \\
-q_{2} & q_{1} & q_{0}-1
\end{array}\right) \Omega \\
& =B(q) \Omega
\end{aligned}
$$

Theorem 1. Let $\alpha$ be a positive constant and $Q$ is any positive definite symmetric matrix. If the desired angular velocity is given by

$$
\Omega_{d}=\alpha I B(q)^{T} Q q
$$

Then, under the stated assumptions and conditions, the overall quaternion system will be exponentially stable. Furthermore, the desired quaternion regulation settling time can be obtained by choosing a suitable value of $\alpha$.

Proof: For simplicity, we will consider $Q=2 I$ in Equation (19) so that

$$
\Omega_{d}=-\alpha \bar{q}
$$

Consider the stable unforced system

$$
\begin{aligned}
\dot{\tilde{q}} & =N(\tilde{q}, \Omega) \\
& =N(\tilde{q}, 0)=0
\end{aligned}
$$

Substituting the value of $\Omega_{d}$ from (20) we have

$$
\dot{\tilde{q}}=-B(q) \alpha \bar{q}
$$

Defining

$$
V=\frac{1}{2}(\tilde{q})^{T} \tilde{q}=1-q_{0}
$$

With substitutions from (8) and (20), we have

$$
\dot{V}=-\dot{q}_{0}=-\frac{1}{2} \bar{q}^{T} \alpha_{i}(\bar{q})
$$

Using the property of quaternion representation [12] that $\bar{q}^{T} \bar{q}+q_{0}^{2}=1$, and since $0 \leq q_{0} \leq 1$, we get

$$
\begin{aligned}
\dot{V} & =-\frac{1}{2} \alpha\left(1+q_{0}\right) V \\
& <0
\end{aligned}
$$

which shows that for the desired input the system is input-tostate stable. This means $\lim _{t \rightarrow \infty} \tilde{q}=0$ and from definition of $\tilde{q}$ we can conclude $\lim _{t \rightarrow \infty} q=q_{e}$.

According to the treatment given in [10], it can be shown that the system is asymptotically stable. For exponential stability; substituting (20) into (8) we have

$$
\begin{gathered}
\dot{q_{0}}=\frac{1}{2} \alpha\left(1-q_{0}^{2}\right) \\
\dot{\bar{q}}=-\frac{1}{2} \alpha q_{0} \bar{q}
\end{gathered}
$$

The time response of $q(t)$, by solving these differential equations can be found as

$$
\begin{gathered}
q_{0}(t)=1-2 c_{1} \frac{e^{-\alpha t}}{1+c_{1} e^{-\alpha t}} \\
\bar{q}(t)=\frac{1+c_{1}}{1+c_{1} e^{-\alpha t}} e^{-0.5 \alpha t} q(0)
\end{gathered}
$$

where $c_{1}$ can be defined as $c_{1}=\frac{1-q_{0}(0)}{1+q_{0}(0)}$. From (21) and (22) we can conclude that the quaternion system (8) is exponentially stable [10]. Also from (21) and (22), it can be seen that the parameter $\alpha$ is related to the settling time of the quaternion regulation and according to definition of the 
regulation settling time $t_{q}$ in [11], this relationship is given as

$$
t_{q}=\frac{4.6}{0.5 \alpha}=\frac{9.2}{\alpha}
$$

2) Desired Angular Velocity Tracking: In the next step, we design $\tau_{a}$ such that it makes the angular velocity $\Omega$, asymptotically follows the desired angular velocity (20). The angular velocity tracking error can be described as $\tilde{\Omega}=\Omega-\Omega_{d}$. Assume that

$$
\dot{\tilde{\Omega}}=-\lambda f(\tilde{\Omega})
$$

in which $\lambda$ is a positive constant and $f(\tilde{\Omega})$ is any function of $\tilde{\Omega}$ which satisfies

$$
\begin{array}{cc}
\tilde{\Omega} f(\tilde{\Omega})>0 & \tilde{\Omega} \neq 0 \\
f(\tilde{\Omega})=0 & \tilde{\Omega}=0
\end{array}
$$

Defining the Lyapunov function candidate as

$$
V=\frac{1}{2}(\tilde{\Omega})^{T} \tilde{\Omega}
$$

The time derivative of $V$ while considering (24) is

$$
\begin{aligned}
\dot{V} & =-\lambda \tilde{\Omega} f(\tilde{\Omega}) \\
& <0
\end{aligned}
$$

which shows that $\lim _{t \rightarrow \infty} \tilde{\Omega}=0$ and subsequently, we have $\lim _{t \rightarrow \infty} \Omega=\Omega_{d}$.

A model-dependent control law, $\tau_{a}$ can now be designed as

$$
\tau_{a}=s(\Omega) I_{f} \Omega+G_{a}+I_{f} \dot{\Omega}
$$

Using the definition of $\tilde{\Omega}$, we have that $\dot{\Omega}=\dot{\tilde{\Omega}}+\dot{\Omega_{d}}$, which with respect to (24) gives

$$
\tau_{a}=s(\Omega) I_{f} \Omega+G_{a}-\lambda I_{f} f(\tilde{\Omega})+I_{f} \dot{\Omega_{d}}
$$

From (20), we have $\dot{\Omega}_{d}=J(q) \dot{q}$, where $J(q)$ is Jacobian matrix of $\Omega_{d}$ as given by

$$
J(q)=\left(\begin{array}{cccc}
0 & -\alpha_{1} & 0 & 0 \\
0 & 0 & -\alpha_{2} & 0 \\
0 & 0 & 0 & -\alpha_{3}
\end{array}\right)
$$

Finally from (15) we get

$$
\dot{\Omega_{d}}=J(q) F(\Omega) q
$$

which yields

$$
\tau_{a}=s(\Omega) I_{f} \Omega+G_{a}-\lambda I_{f} f(\tilde{\Omega})+I_{f} J(q) F(\Omega) q
$$

Remark 1. As an example, one of the functions that can satisfy Equations (25) and (26) is

$$
f(\tilde{\Omega})=\operatorname{sat}(\tilde{\Omega})=\left\{\begin{aligned}
\tilde{\Omega} & |\tilde{\Omega}|<a \\
\operatorname{sgn}(\tilde{\Omega}) & |\tilde{\Omega}| \geq a
\end{aligned}\right.
$$

Where the positive constant $a$ is the width of the boundary layer of the saturation function.

Remark 2. The control law (29) will ensure the asymptotic stability of the quadrotor if and only if the regulation of $\Omega$ to its equilibrium point (zero) is faster than regulation of $q$ to $q_{e}$ which means that

$$
t_{\Omega}<t_{q}
$$

Here, the angular velocity tracking error settling time $t_{\Omega}$ approximately is:

$$
t_{\Omega}=\frac{a+\alpha}{\lambda \alpha}
$$

Notice that the boundary layer width a, has to be sufficiently small such that it is in the angular velocity settling range. Also the control law (29) with respect to the airframe inertia uncertainties $\Delta I_{f}$ is robustly stable if the angular velocity tracking parameter $\lambda$ is

$$
\lambda>\lambda_{0}=a\left(a+\frac{1}{2}\|J\|_{\infty}\right) \frac{\delta}{\sigma_{\min }\left(I_{f 0}\right)}
$$

where $I_{f 0}$ is nominal value of airframe's inertia matrix and $\Delta I_{f} \in\left\{y \mid\|y\|_{\infty} \leq \delta\right\}$.

It is important to point out here that one can simply design a model-independent control law as

$$
\begin{aligned}
\tau_{a} & =\dot{\Omega} \\
& =-\lambda f(\tilde{\Omega})+J(q) F(\Omega) q
\end{aligned}
$$

Further, with both the control laws of (29) and (32), the regulation problem of attitude angles to their desired values (i.e. at zero as in hovering case) results in an exponentially stable system, and the regulation settling time is a function of $\alpha$.

\section{B. Designing the Rotor Torque $\tau_{i}$}

Having achieved the task of designing control torque (model dependent or model independent), we now proceed to designing $\tau_{i}$ such that the angular speed of the rotors $\left(w_{i}\right.$ 's), follow the desired angular speeds generated by our designed control torque $\tau_{a}=\left(\tau_{\phi}, \tau_{\theta}, \tau_{\psi}\right)^{T}$. From Equations (1)-(3) and (7), we find the desired angular speeds as

$$
\begin{aligned}
\left(\begin{array}{c}
w_{d_{1}}^{2} \\
w_{d_{2}}^{2} \\
w_{d_{3}}^{2} \\
w_{d_{4}}^{2}
\end{array}\right) & =\left(\begin{array}{cccc}
0 & b d & 0 & -b d \\
b d & 0 & -b d & 0 \\
l & -l & l & -l \\
b & b & b & b
\end{array}\right)^{-1}\left(\begin{array}{c}
\tau_{\phi} \\
\tau_{\theta} \\
\tau_{\psi} \\
T
\end{array}\right) \\
& =A^{-1}\left(\begin{array}{c}
\tau_{\phi} \\
\tau_{\theta} \\
\tau_{\psi} \\
T
\end{array}\right)
\end{aligned}
$$

in which the parameters $b, d$ and $l>0$ are assumed to be positive, in order to ascertain that the matrix $A$ remains nonsingular.

Tracking error of angular speed of rotor's can be described as $\tilde{w}_{i}=w_{i}-w_{d_{i}}$. For making the angular speeds of rotors asymptotically approach their respective $w_{d_{i}}$ 's, assume that

$$
\dot{\tilde{w}}_{i}=-h f\left(\tilde{w}_{i}\right)
$$

in which $h$ is a positive constant and $f\left(\tilde{w}_{i}\right)$ is any function of $\tilde{w}_{i}$ that satisfies (25) and (26) by replacing $\tilde{\Omega}$ with $\tilde{w}_{i}$. Defining the Lyapunov function candidate as 


$$
V=\frac{1}{2}\left(\tilde{w}_{i}\right)^{T} \tilde{w}_{i}
$$

TABLE I

The time derivative of $V$ while considering (34) is

$$
\begin{aligned}
\dot{V} & =-h \tilde{w}_{i} f\left(\tilde{w}_{i}\right) \\
& <0
\end{aligned}
$$

which shows that $\lim _{t \rightarrow \infty} \tilde{w}_{i}=0$ and subsequently, we have $\lim _{t \rightarrow \infty} w_{i}=w_{d_{i}}$.

We can simply define $\tau_{i}$ with respect to (10), as

$$
\tau_{i}=Q_{i}+I_{r} \dot{w}_{i}
$$

which can be written as

$$
\tau_{i}=Q_{i}+I_{r} \dot{w_{d_{i}}}-h I_{r} f\left(\tilde{w}_{i}\right)
$$

One of the functions that can satisfy the conditions is

$$
f\left(\tilde{w}_{i}\right)=\operatorname{sat}\left(\tilde{w}_{i}\right)=\left\{\begin{aligned}
\tilde{w}_{i} & \left|\tilde{w}_{i}\right|<a \\
\operatorname{sgn}\left(\tilde{w}_{i}\right) & \left|\tilde{w}_{i}\right| \geq a
\end{aligned}\right.
$$

Notice that in Equation (35), one of the possible means of finding $w_{d_{i}}$ can be by using the dirty derivative filter [4];

$$
\dot{w}_{d_{i}}=\frac{s}{1+T_{f} s} w_{d_{i}}
$$

\section{Simulation Results}

In order to ascertaining the performance of the quadrotor using the proposed control algorithm, we consider a quadrotor with dynamical parameters as listed in Table 1. Using Equation (23), the desired regulation settling time (attitude angles settling time) can give us the required parameters for our control law. Notice that, there is a compromise between choosing a small value of $t_{q}$ and having large peak value for the angular velocity $\Omega$, since with replacing the control law (29) or (32) in (9), we will find that peak value of the angular velocity $\Omega$ would be a function of $\alpha, \lambda$ and $a$. In other words, if we decrease $t_{q}$, we have increased $\alpha$ and decreased $\lambda$, which will increase the peak value. Furthermore, choosing $a$ as small as possible will cause to smaller peak values. In this paper we have considered the value of $a$ as:

$$
a=0.02 \alpha
$$

Simulation 1 (Fig. 2 and Fig. 3) shows the performance of the control law (29) for desired regulation settling time $\left(t_{q}\right)$ $0.2 s$. From (23) and (37) respectively we will get $\alpha=46$ and $a=0.92$. One can choose $t_{\Omega}=0.1$ and then from (31), $\lambda=510$. Simulation 2 (Fig. 4 and Fig. 5) shows the performance of the control law (32) for the previous values.

Consider the initial angles as $\phi=-25^{\circ}, \theta=30^{\circ}, \psi=$ $-10^{\circ}$, gain $h_{i}, i \epsilon\{1,2,3,4\}$ as $0.002, T=1.5 N$ and $T_{f}=0.008$ (cutoff frequency of $20 \mathrm{~Hz}$ ). In Simulations 1 and 2 , the desired situation of quad rotor is in hovering. Notice that, performance of both the control laws is nearly equivalent. Trajectories of angular speed of rotors for the system described in Simulation 1 have been depicted in Fig. 6. In Simulation 3 (Fig. 7 and Fig. 8) the system is to be regulated to $\phi_{r}=10^{\circ}, \theta_{r}=-15^{\circ}, \psi_{r}=5^{\circ}$. As before, performance of model-independent control law (32) is same as model-dependent control law (29). Trajectories of angular speed of rotors are shown in Fig. 9.

\begin{tabular}{|c|l|}
\hline $\begin{array}{c}\text { Dynamical } \\
\text { Parameter }\end{array}$ & $\begin{array}{l}\text { Considered } \\
\text { Value }\end{array}$ \\
\hline $\mathrm{d}$ & $0.225 \mathrm{~m}$ \\
$I_{r}$ & $3.4 \times 10^{-5} \mathrm{~kg} \cdot \mathrm{m}^{2}$ \\
$I_{f_{\phi}}$ & $4.9 \times 10^{-3} \mathrm{~kg} \cdot \mathrm{m}^{2}$ \\
$I_{f_{\theta}}$ & $4.9 \times 10^{-3} \mathrm{~kg} \cdot \mathrm{m}^{2}$ \\
$I_{f_{\psi}}$ & $8.8 \times 10^{-3} \mathrm{~kg} \cdot \mathrm{m}^{2}$ \\
$b$ & $2.9 \times 10^{-5}$ \\
$l$ & $1.1 \times 10^{-6}$ \\
\hline
\end{tabular}

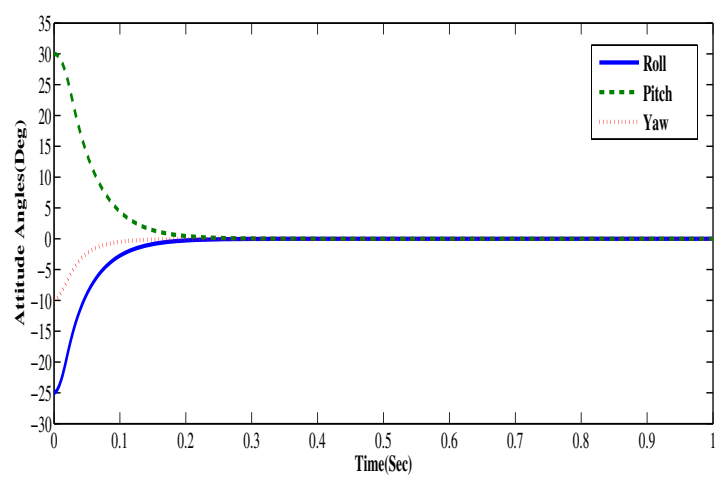

Fig. 2. Simulation 1: Attitude Angles with Controller (29)

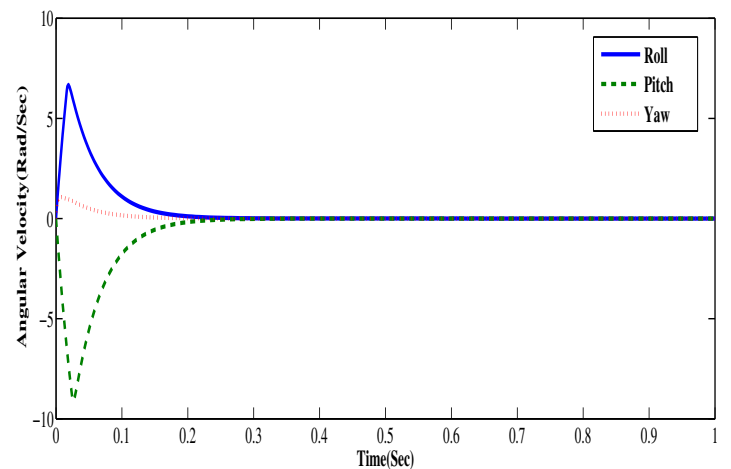

Fig. 3. Simulation 1: Angular Velocities with Controller (29)

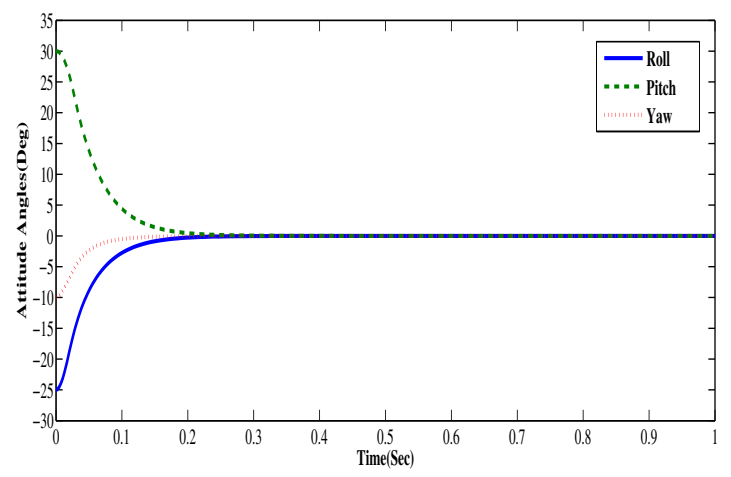

Fig. 4. Simulation 2: Attitude Angles with Controller (32) 


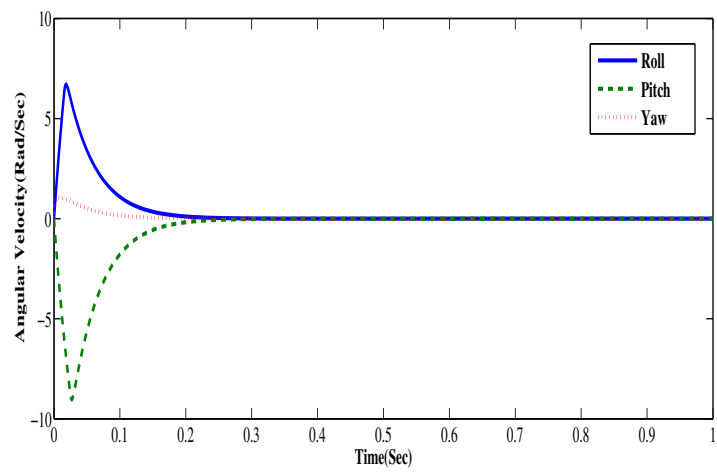

Fig. 5. Simulation 2: Angular Velocities with Controller (32)

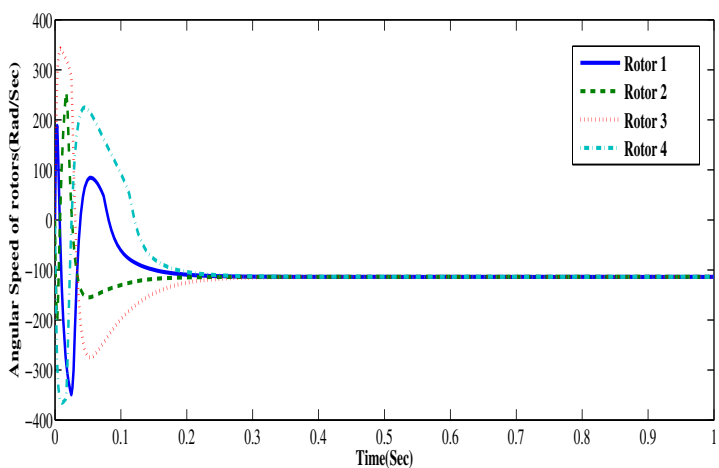

Fig. 6. Simulation 1: Angular Speed of Rotors

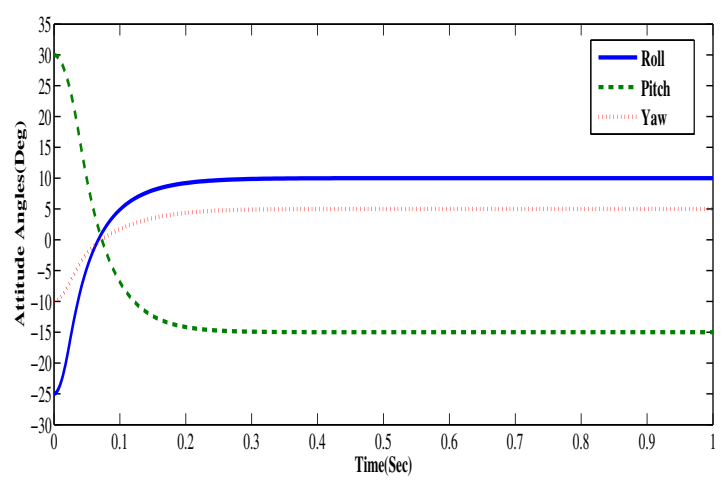

Fig. 7. Simulation 3: Attitude Angles with Controller (29)

\section{CONCLUSIONS}

We presented a novel control approach to obtain asymptotic attitude stability of a quadrotor as a representative of VTOL-UAVs. Based upon the quaternion representation, we defined a robust control law for attitude control of the quadrotor in which the regulation of attitude angles to their desired values is shown to be exponentially stable, and that the desired settling values can be adjusted by the operator. In the proposed methodology, the tracking of desired angular velocity is shown to be asymptotically stable. The performance of the proposed control design has been ascertained using Simulation.

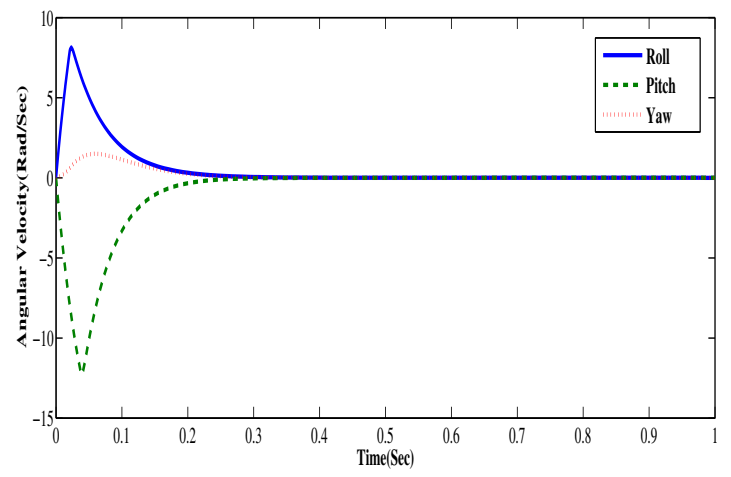

Fig. 8. Simulation 3: Angular Velocities with Controller (29)

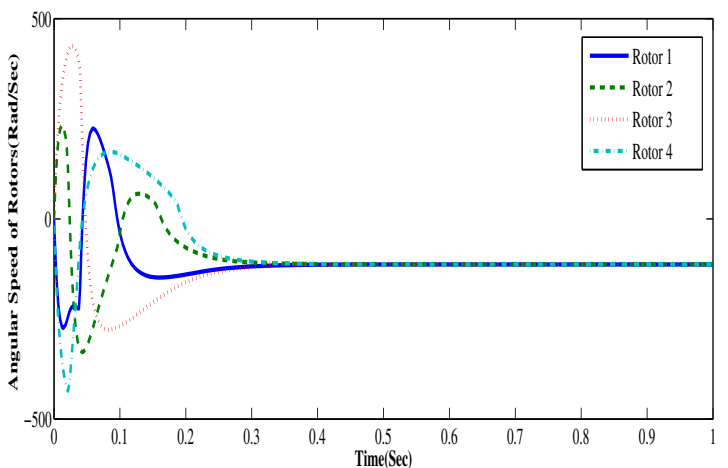

Fig. 9. Simulation 3: Angular Speed of Rotors

\section{REFERENCES}

[1] A. Abdessameud and A. Tayebi, Formation Stabilization of VTOL UAVs Subject to Communication Delays, In proc. of the 49th IEEE CDC, Atlanta, GA, USA, 2010.

[2] J. T-Y. Wen and K. Kreutz-Delgado, The Attitude Control Problem, IEEE Transaction on Automatic Control, Vol. 36, No. 10, 1991.

[3] T. Hamel, R. Mahony, R. Lozano and J. Ostrowski, Dynamic Modelling and Configuration Stabilization for an X4-Flyer, In Proc. of IFAC World Congress, Barcelona, Spain, 2002.

[4] A. Tayebi and S. McGilvray, Attitude Stabilization of a VTOL Quadrotor Aircraft, IEEE Transactions on Control Systems Technology, Vol. 14, No. 3, 2006.

[5] P. Castillo, P. Albertos, P. Garcia, and R. Lozano, Simple Real-time Attitude Stabilization of a Quad-rotor Aircraft with Bounded Signals, In proc. of the 45th IEEE CDC, San Diego, CA, USA, 2006.

[6] A. Tayebi, S. McGilvray, A. Roberts, and M. Moallem, Attitude estimation and stabilization of a rigid body using low-cost sensors, In proc. of the 46th IEEE CDC, New Orleans, LA, USA, 2007.

[7] A. Tayebi, A. Roberts and A. Benallegue, Inertial Measurements Based Dynamic Attitude Estimation and Velocity-Free Attitude Stabilization, In Proc. of ACC, San Francisco, CA, USA, 2011.

[8] R. Mahony and T. Hamel, Adaptive compensation of aerodynamic effects during takeoff and landing maneuvers for a scale model autonomous helicopter, European Journal of Control, Vol.7, 2001.

[9] R. M. Murray, Z. Li and S. S. Sastry , A Mathematical introduction to Robotic Manipulation, CRC Press, 1994.

[10] H. K. Khalil, Nonlinear Systems, 3rd ed. Prentice Hall, 2002.

[11] J. Lu and B. Wie, Nonlinear Quaternion Feedback Control for Spacecraft via Angular Velocity Shaping, In proc. of ACC, Baltimore, MD, USA, 1994.

[12] S. M. Joshi, A. G. Kelkar and J. T-Y. Wen, Robust Attitude Stabilization of Spacecraft Using Nonlinear Quaternion Feedback, IEEE Transactions on Automatic Control, Vol. 40, No. 10, 1995. 